\title{
EXPLOTACIÓN DEL RECURSO BIDIRECCIONAL DE CANAL INALÁMBRICO APLICADO A CONFERENCIAS TRADUCIDAS
}

\section{RESOURCE EXPLOITATION OF WIRELESS CHANNEL APPLIED TO TRANSLATION CONFERENCE}

\author{
Yesica Beltrán-Gómez ${ }^{1} \quad$ Rafael Linero-Ramos ${ }^{2} \quad$ Jorge Gómez-Rojas ${ }^{3}$
}

Forma de citar: BELTRÁN GÓMEZ Yesica, LINERO-RAMOS Rafael, GÓMEZ-ROJAS Jorge. Explotación del recurso bidireccional de canal inalámbrico aplicado a conferencias traducidas. Respuestas. 2013; 18(2): 96-101

Recibido:

Marzo 25 de 2013

Aceptado:

Julio 30 de 2013

\section{RESUMEN}

Este artículo presenta una solución tecnológica propuesta para una deficiencia identificada en los sistemas de telecomunicaciones empleados para la realización de conferencias en las cuales el ponente y el auditorio difieren en idioma de habla. La propuesta está enfocada a satisfacer la necesidad al momento de realizar intervenciones por parte del auditorio, mediante la implementación de un sistema inalámbrico en modo de transmisión full-dúplex, y de una central de traducción y moderamiento con el fin de lograr una comunicación más efectiva entre el ponente y el auditorio, que la comunicación lograda con los dispositivos inalámbricos en modo de transmisión unidireccional que actualmente se conocen para este tipo de aplicaciones.

Palabras clave: ZigBee, radiocomunicación, Frecuencia Modulada FM, bidireccional.

\section{ABSTRACT}

This paper presents a proposed technological solution to a deficiency identified in the telecommunication systems used for holding conferences in which the speaker and the audience differ in language speech. The proposal is focused to meet the need to realize interventions by the auditory, by means of wireless systems in full duplex mode, and a translating core to achieve a communications more effective between speaker and audience, that the communications achieved with the wireless devices in unidirectional transmission mode that currently known for this applications types.

Keywords: ZigBee, radiocommunication, Frequency Modulated FM, bidirectional. 


\section{INTRODUCCIÓN}

La utilización de las comunicaciones en los avances tecnológicos para la realización de conferencias en las cuales el ponente y el auditorio difieran en idioma de habla se ha logrado mediante sistemas inalámbricos que comunican al ponente con una central de traducción y desde ésta, la información suministrada por el ponente se transmite al auditorio en el idioma deseado.

Se crea la necesidad de este tipo de comunicaciones con las expansiones territoriales y de los mercados de diferentes países del mundo, y con el fin de suplir dicha necesidad es fundada en 1953 la Asociación Internacional de Intérpretes de Conferencias (AIIC) siendo esta la única asociación de intérpretes de conferencias profesionales de ámbito mundial [1].

Este tipo de comunicaciones son requeridos en las Naciones Unidas, la Unión Europea, las administraciones públicas, empresas privadas, organismos científicos y de investigación y organizadores profesionales de congresos, entre otros. Actualmente son utilizados para este tipo de comunicación sistemas con dispositivos inalámbricos en modo de transmisión unidireccional como el sistema inalámbrico para guías turísticos, traductores y docentes Okayo diseñado por la compañía E-J Electronics Co., Ltd. [2], mediante los cuales solo es posible establecer una comunicación en la cual la central de traducción transmite hacia el auditorio la información en el idioma deseado, que previamente el ponente de la conferencia ha transmitido hacia esta central.

Otro dispositivo empleado en transmisiones bidireccionales es el BITX el cual es un diseño de transceptor para la banda de radioaficionados de $20 \mathrm{~m}$, en modulación banda lateral única, creado por el ingeniero Ashhar Farhan [3]; está destinado a los radioaficionados que desean construir su propio equipo de transmisión.

El BITX ha sido utilizado por radioaficionados de muchos países: Francia, Inglaterra, Estados Unidos, Singapur, Nueva Zelandia, Brasil y la India, entre otros. Los comentarios de los constructores y de sus interlocutores son elogiosos. Los objetivos de costo y disponibilidad fueron alcanzados.

La implementación del sistema propuesto se hizo con el fin de lograr una comunicación más efectiva entre un ponente y un auditorio que difieren en idioma de habla, se desarrolló un dispositivo bidireccional el cual además de la recepción de la información proveniente de una central de traducción, también permite mediante un canal de retorno, la comunicación hacia la central, de la intervención del auditorio de la conferencia y los comunica al ponente, teniendo en cuenta que en la central se tendrá un sistema de moderamiento para ceder la palabra, como se observa en la figura 1 .

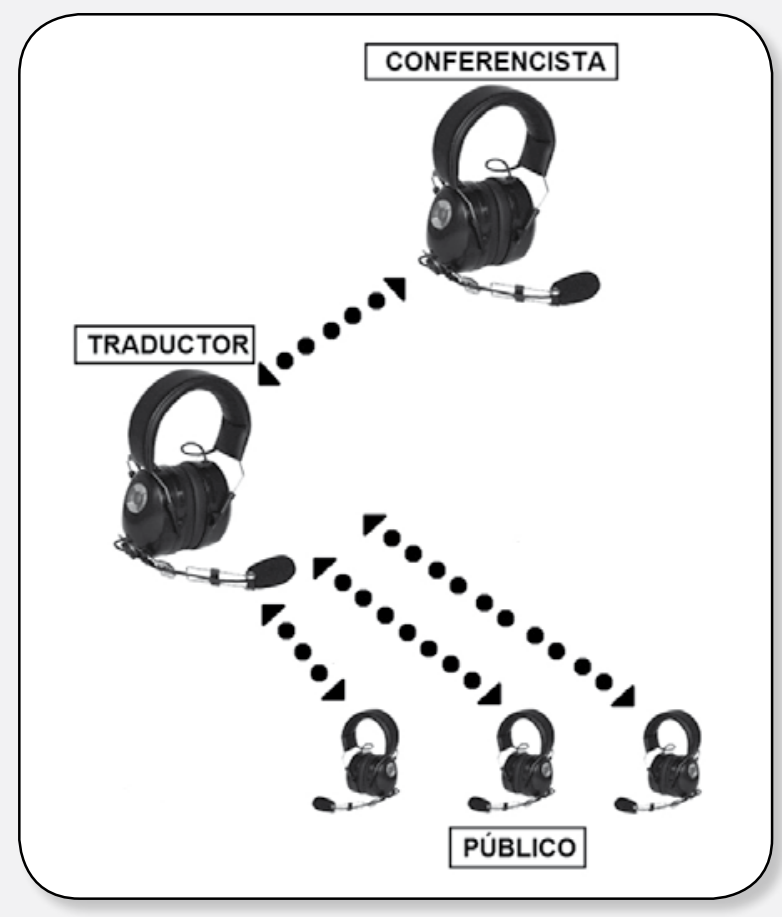

Figura 1. Sistema inalámbrico Full-Dúplex.

\section{METODOLOGÍA} ISSN 0122-820X

PP: 96-101

Para el desarrollo de este proyecto se empleó un plan de trabajo en el cual se utilizó la Estructura para Desglose de Tarea (Work Breakdown Structure, WSB) mediante la cual se dividió el trabajo a realizar de la siguiente forma: a partir de los objetivos se generan 
No. 2

Junio - Diciembre 2013 ISSN 0122-820X

PP: 96-101

actividades, y las actividades se desglosan en tareas.

En primera instancia se documentó información de dispositivos con características y funcionamiento similar al que se desarrolló, se recolectó información aplicando una entrevista, en la cual se plantearon una serie de preguntas para evaluar el nivel de satisfacción de la población seleccionada con respecto a sistemas actuales de traducción de conferencias.

Como segunda fase en la construcción de este proyecto se planeó el diseño de la etapa de control y moderamiento; se definieron los objetivos del diseño, que son los que delimitan los requerimientos de la etapa que se está construyendo, se estableció la lógica mediante la cual la central de moderamiento controlará el uso de la palabra, se seleccionaron los criterios de diseño: en los cuales se plantearon las premisas para cumplir con los objetivos del diseño, se desarrolló la lógica programable y los cálculos para hallar los valores de cada elemento del circuito, y así finalmente obtener el diseño de la etapa de control y moderamiento, cumpliendo con los objetivos del diseño.

La tercera fase es la correspondiente al diseño de la etapa de comunicaciones, luego de todo esto continúa la fase correspondiente a la simulación del sistema diseñado completo (dispositivos-central), y la última fase correspondió a la implementación el prototipo para verificar y evaluar el funcionamiento del sistema.

Los equipos utilizados fueron los dispositivos XBee basados en ZigBee para la etapa de control y moderamiento y los dispositivos Laipac RF900DV para la etapa de comunicación.

ZigBee es un conjunto de protocolos de alto nivel de comunicación inalámbrica [4], basada en el estándar IEEE 802.15.4 de redes inalámbricas de área personal (WPAN, Wireless Personal Area Network) [5]. Su objetivo son las aplicaciones que requieren comunicaciones seguras con baja tasa de envío de datos y maximización de la vida útil de sus baterías [6].
Las principales características de los módulos XBee son:

- Buen Alcance: hasta 300ft (100 mts) en línea vista.

- 9 entradas/salidas con entradas analógicas y digitales.

- Bajo consumo $<50 \mathrm{~mA}$ cuando están en funcionamiento y $<10 u A$ cuando están en modo sleep.

- Interfaz serial.

- 65,000 direcciones para cada uno de los 16 canales disponibles. Se pueden tener muchos de estos dispositivos en una misma red.

- Fáciles de integrar.

- Bajo costo [7].

Los módulos Laipac RF900DV son transceptores RF de alto rendimiento diseñados para aplicaciones como sistema de intercomunicación, seguridad, telemetría, un módem inalámbrico, beeper personal, red local inalámbrica, dispositivos portátiles inalámbricos, entre otros. El rango es de más de 1200 pies y puede enviar datos con una velocidad de 28.8Kbps o audio de dúplex completo con 16 canales disponibles para seleccionar [8].

\section{DESARROLLO}

\subsection{Control y moderamiento}

Para el diseño y la implementación de la etapa de control y moderamiento se escogió la tecnología ZigBee, mediante el uso de los dispositivos XBee los cuales integran un transmisor y receptor de ZigBee y un procesador en un mismo módulo, lo que le permite a los usuarios desarrollar aplicaciones de manera rápida y sencilla [7].

Los módulos XBee son programados en la configuración punto-multipunto, de esta manera el modulo configurado como coordinador es 
asignado a la central de traducción y moderamiento junto con el dispositivo emisorreceptor del traductor, y los demás módulos son configurados como dispositivos finales y asignados a los usuarios del sistema [9].

El circuito completo diseñado e implementado para la central de control y moderamiento consta de los siguientes elementos:

- un microcontrolador $18 \mathrm{~F} 4455$

- un modulo Xbee

- una LCD 2X16

- reguladores de voltaje

Los reguladores de voltaje son utilizados debido a que el circuito es alimentado con una batería de $9 \mathrm{~V}$, así que se debe regular el voltaje tanto para el microcontrolador como para el $\mathrm{XBee}$ con $5 \mathrm{~V}$ y 3.3V respectivamente.

La LCD es utilizada para observar el estado en que se encuentra el sistema; cuando está inicializando, cuando está en modo de transmisión y para mostrar el usuario a quien se le asigna la palabra, por ejemplo, si el usuario \# 1 solicita el uso de la palabra y el sistema se lo asigna, en la pantalla de la LCD se va observar: "Palabra asignada usuario 1".

El módulo XBee es parte fundamental de esta etapa ya que permite implementar la red inalámbrica para el control de la palabra. El módulo XBee configurado como coordinador transmite un dato a los usuarios end device para asociar todos los dispositivos que se encuentran en la misma PAN ID y para indicar que se encuentra preparado para recibir los pedidos de la palabra; el usuario que desea solicitar la palabra oprime un pulsador y con este se envía un dato de identificación único para cada usuario, cuando el XBee coordinador recibe el dato lo retorna y de esta manera activa el transmisor del usuario para que haga su intervención. Una vez finalizada la intervención de cada usuario el coordinador desactiva el transmisor que había sido activado y vuelve a quedar preparado para seguir recibiendo los pedidos de la palabra.
El microcontrolador es utilizado para enviar los datos a imprimir en la LCD, además envía el dato de autorización para el pedido de la palabra al XBee, por medio del puerto Usart. Evalúa el dato que recibe de los dispositivos end device, retorna el dato al XBee y restablece nuevamente el puerto.

El circuito implementado para los usuarios del sistema es similar al de la central, aunque tiene algunas variaciones tales como: No cuenta con una LCD, en lugar de ésta el circuito de los usuarios tiene un led que se encenderá cuando se le asigne la palabra y un pin del PIC controla el encendido y apagado del transmisor de audio. El microcontrolador envía un dato que lo identifica como el usuario " $\mathrm{x}$ ", este dato es enviado y recibido mediante los módulos XBee y la central lo retorna. El usuario recibe el dato retornado y lo compara, si es igual al que él envió se activa el transmisor y se asigna la palabra.

Cabe resaltar que para la programación general del sistema, el dato que recibe la central y retorna al usuario es el primer dato recibido por el XBee, una vez recibe dicho dato el traductor anuncia mediante un mensaje de voz a cual usuario se le asignó la palabra, de esta manera la central no toma en cuenta los posibles siguientes datos recibidos. Cuando el primer usuario termina de hacer uso de la palabra, el traductor envía nuevamente un mensaje de voz anunciando que los demás usuarios que desean solicitar la palabra ya pueden hacerlo y la central se encuentra preparada para recibir otro pedido, nuevamente los usuarios que desean intervenir deben hacer la solicitud.

\subsection{Comunicación}

Para la etapa de comunicaciones se escogieron los módulos de transmisión de Laipac RF900DV. En este sistema, se utiliza un elemento base que puede comunicarse con varios elementos remotos. La selección del canal se hace a través de 4 pines de entrada [8].

El modulo transceptor trabaja en el rango de frecuencia de $900 \mathrm{MHz}$, el canal se puede escoger como se muestra en la tabla 1 , se hace la selección aleatoriamente que en nuestro caso
Junio - Diciembre 2013 ISSN 0122-820X

PP: 96-101 
No. 2

Junio - Diciembre 2013 ISSN 0122-820X

PP: 96-101

fue escogido el canal 6, configurando los pines D0, D1, D2 y D3 en 1010 respectivamente.

Finalmente lo que se hace es la adecuación de estas dos etapas, la cual es sencilla; el microcontrolador hace la selección del canal mediante 4 pines del puerto $\mathrm{D}$, y además cuando recibe la señal de autorización para la palabra, es decir, cuando se enciende el led de indicación de que ya puede hacer uso de la palabra, el transmisor se activa. Cuando no se tiene el uso de la palabra la alimentación del transmisor del módulo se encuentra interrumpida.

Tabla 1: Tabla para la selección del Canal [8]

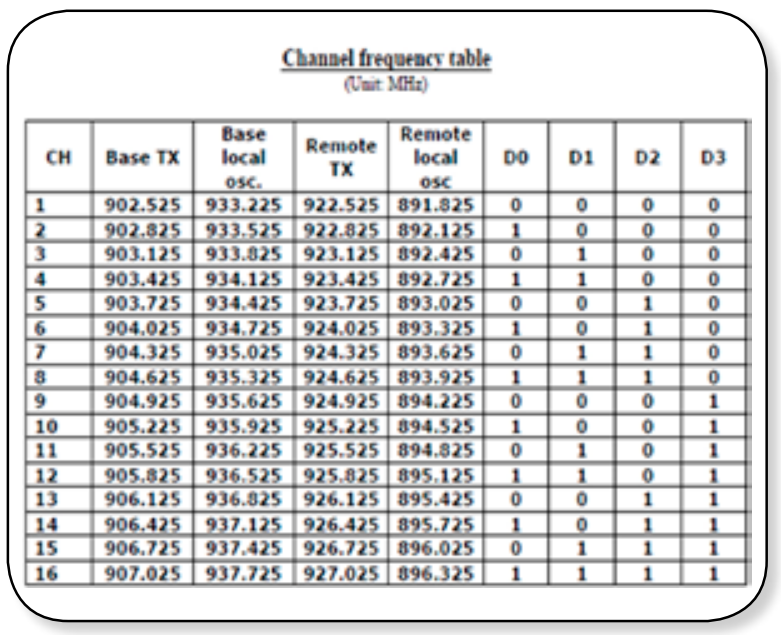

El circuito transceptor del traductor tendrá habilitado durante todo el tiempo tanto el transmisor como el receptor ya que se encontrara en constante uso de la palabra, la descripción hecha anteriormente donde se interrumpe y se activa el transmisor solo aplica para los usuarios finales del sistema.

\section{CONCLUSIONES}

Se hace uso de una tecnología diferente para cada etapa ya que los módulos XBee permiten la realización de una red punto-multipunto enviando información de manera controlada debido a que cada dispositivo cuenta con un serial único que lo identifica y además se encuentra fundamentada en un protocolo de comunicaciones inalámbrico basado en el estándar de comunicaciones para redes inalámbricas IEEE 802.15.4. Y son usados los módulos
RF900DV con un sistema de modulación FM ideales para el envío de voz en modo de transmisión full-duplex y con esto poder lograr el desarrollo e integración de las dos etapas del proyecto.

A lo largo del desarrollo del proyecto se exploraron procesos de digitalización de voz para realizar la transmisión de ésta por medio de dispositivos XBee, y a pesar de haber logrado transmisiones de voz con baja calidad, solo fue conseguido en conexiones punto a punto, pero esto no arrojó resultados satisfactorios al momento de implementar la red requerida en la aplicación propuesta para el uso del sistema desarrollado, debido a que los módulos XBee trabajan con envío de paquetes de acuse de recibido al momento de establecer comunicaciones en redes, por lo que realiza envíos de paquetes de datos de 8 bytes de forma intermitente. Esta forma de envío de datos es la que no permite la transmisión de audio de forma adecuada en una red de dispositivos XBee, por esta razón se optó por emplear un sistema de modulación FM para el envío de inalámbrico de voz.

La integración de las dos etapas que conforman el proyecto es considerada de gran importancia, ya que finalmente el objetivo completo se logró llevando a cabo esta actividad y es fundamental que cada etapa funcione correctamente tanto independiente como conjuntamente.

Luego de la realización de las pruebas necesarias para verificar totalmente el funcionamiento del sistema fueron obtenidos resultados satisfactorios. Para emplear el sistema desarrollado en este proyecto en auditorios que demanden un alcance mayor para la transmisión de datos se pueden remplazar los módulos XBee por módulos XBee-PRO que poseen alcance con línea de vista hasta de 1500 metros.

En cuanto a la simultaneidad al momento de solicitar el uso de la palabra el sistema desarrollado se comporta de manera satisfactoria autorizando al usuario que primero envíe la señal de solicitud, la diferencia porcentual arrojada en las pruebas realizadas, al ser pe- 
queña, muestra que esta es causada por la diferencia mínima de tiempo existente entre un humano y otro al realizar la acción de pulsar para solicitar el uso de la palabra.

\section{REFERENCIAS}

[1] Asociación Española de Profesionales de la Interpretación de Conferencias, Espaiic, "Aiic en España es Espaiic.” [En línea]. Disponible en: http://espaiic.es/ descargas/folleto_A4_espaiic-nuevo. pdf. [Acceso: 20-Agosto-2012].

[2] Audioguiarte Servicios Culturales S.L., "Equipos Okayo: Audioguías de grupo." [En línea]. Disponible en: http://www. audioguiarte.com/equipos/okayo_general.pdf. [Acceso: 26-Septiembre-2011].

[3] PhoneStack Technologies Pvt. Ltd., "BITX - An easy to build 6 watts SSB transceiver for $14 \mathrm{MHz}$." [En línea]. Disponible en: http://www.phonestack. com/farhan/bitx.html. [Acceso: 26-Septiembre-2011].

[4] ZigBee Alliance, "ZigBee Technology." [En línea]. Disponible en: http://zigbee. org/About/AboutTechnology/ZigBeeTechnology.aspx. [Acceso: 15-Julio-2012].

[5] IEEE Standard for Information Technology - Telecommunications and Information Exchange Between Systems - Local and Metropolitan Area Networks - Specific requirements Part 15.4: Wireless Medium Access Control (MAC) and Physical Layer (PHY) Specifications for Low-Rate Wireless Personal Area Networks (WPANs), IEEE Std 802.15.4, 2006.

[6] P. Baronti, P. Pillai, V. W. C. Chook, S. Chessa, A. Gotta, and Y. F. Hu, "Wireless sensor networks: A survey on the state of the art and the 802.15.4 and ZigBee standards," Computer Communications., vol. 30, no. 7, pp. 1655-
1695, 2007.

Junio - Diciembre 2013 ISSN 0122-820X

[7] D. International, "XBee $®$ / XBeePP: 96-101 PRO ${ }^{\circledR}$ ZB RF Modules Digi International Inc.", 2010. [En línea]. Disponible en: http://ftp1.digi.com/support/ documentation/90000976_P.pdf. [Acceso: 15-Julio-2012].

[8] Laipac Technology Inc., "RF900DV Specification Sheet." [En línea]. Disponible en: http://www.laipac.com/Downloads/ Easy/RF900DV-16ch-06-26-2004.pdf. [Acceso: 15-Julio-2012].

[9] J. Lee, Y. Su, and C. Shen, "A comparative study of wireless protocols: Bluetooth, UWB, ZigBee, and Wi-Fi," in Proc. IECON, Taipei, Taiwan, Nov. 2007, pp. 46-51. 